\title{
Relocation of a seafloor transponder-Sustaining the GPS-Acoustic technique
}

\author{
K. L. Gagnon and C. D. Chadwell \\ Marine Physical Lab, Scripps Institution of Oceanography, La Jolla, CA, U.S.A. \\ (Received January 17, 2007; Revised March 6, 2007; Accepted March 8, 2007; Online published June 8, 2007)
}

\begin{abstract}
Rigid seafloor arrays of three to four precision acoustic transponders have been repeatedly positioned with the GPS-Acoustic technique to measure horizontal plate motion. In the event that one transponder becomes inactive, a replacement transponder must be precisely located relative to the existing array. Here we present a technique to determine the geodetic azimuth and baseline between the inactive and replacement transponders. We include three examples of relocations between 2002 and 2003 on the Juan de Fuca plate and near the Peru-Chile trench, which add $\pm 16-29 \mathrm{~mm}$ uncertainty to the GPS-Acoustic estimated position. A simulation of optimal network geometry shows that the relocation's contribution to uncertainty can be as low as $\pm 10 \mathrm{~mm}$.
\end{abstract}

Key words: Seafloor geodesy, GPS-Acoustic.

\section{Introduction}

The Global Positioning System is a powerful geophysical tool, allowing the measurement of subaerial plate motion on the order of millimeters. Through a combination of GPS and acoustics, this capability can be extended to the seafloor. The GPS-Acoustic (GPSA) approach combines campaign style kinematic GPS on a floating platform (ship or buoy) and acoustic signals from the platform to an array of seafloor transponders (Spiess, 1985; Purcell et al., 1991; Spiess et al., 1998; Osada et al., 2003). Each transponder is interrogated from the center of the array, creating uniformly spaced raypaths at the surface, where the sound speed is most variable. The final vector uncertainty of the array is on the order of millimeters when the sound speed is negated in this way (Spiess et al., 1998; Gagnon et al., 2005). The baselines of the seafloor array are held fixed in a least-squares solution so that the horizontal displacement of each transponder reflects horizontal plate motion. If one transponder in the array ceases to function, a replacement is positioned within 3 meters of the inactive. To reference GPSA surveys from previous epochs, the new orientation of the array, or more specifically, the precise offset from the inactive to the replacement transponder must be determined. Here we discuss a method for positioning a replacement transponder in an existing array and present results from three relocations at arrays on the Juan de Fuca plate and continental slope offshore Peru. Also, a simulation of the optimal network geometry is shown that minimizes the influence of observational uncertainties.

\section{Background}

Scripps Institution of Oceanography (SIO) began GPSA campaigns to measure the motion of a rigid transponder array at the northern Cascadia subduction zone from 1994 to

Copyright (c) The Society of Geomagnetism and Earth, Planetary and Space Sciences (SGEPSS); The Seismological Society of Japan; The Volcanological Society of Japan; The Geodetic Society of Japan; The Japanese Society for Planetary Sciences; TERRAPUB
1996 (Spiess et al., 1998). This effort provided a measurement of the Juan de Fuca-North America plate convergence with a repeatability of $\pm 39 \mathrm{~mm}$ east and $\pm 8 \mathrm{~mm}$ north after roughly 30 hours of data collection in each of the three years. Four additional sites were created to investigate various forms of crustal deformation as well as further develop the technique. One array was created on the south Cleft segment of the Juan de Fuca Ridge to investigate plate motion $25 \mathrm{~km}$ east of the spreading axis (Spiess et al., 2000). Another was installed on the Juan de Fuca plate, $150 \mathrm{~km}$ west of Newport, Oregon, to study convergence at the southern Cascadia subduction zone. Two transponder arrays were installed 20 and $50 \mathrm{~km}$ landward of the PeruChile trench to determine the magnitude of horizontal deformation above the subduction zone. After roughly 100 hours of data collection at each array the repeatability was \pm 5-7 mm (Gagnon et al., 2005). Finally, seven transponders were deployed on the slope of the Hilina fault system of Kilauea volcano to study aseismic submarine slumping (Hildebrand et al., 2000; Phillips and Chadwell, 2005).

Several organizations in Japan have also used a combination of GPS and acoustic measurements to study crustal deformation. Tohoku University used the GPSA approach from a towed buoy to measure an array with an uncertainty of $\pm 50 \mathrm{~mm}$ after 48 hours at Kumano-nada in the Nankai trough (Kido et al., 2006). Jointly, the University of Tokyo, Tohoku University, and SIO demonstrated array positioning of $\pm 30 \mathrm{~mm}$ east and $\pm 31 \mathrm{~mm}$ north after roughly 18 hours of GPSA data on the slope of the Hilina fault system in Kilauea (Osada et al., 2003).

The Hydrographic and Oceanographic Department of Japan (JHOD) and the Institute of Industrial Science, University of Tokyo (IIS) have installed over 15 seafloor reference points. At the Japan trench off the Miyagi Prefecture, seven GPSA campaigns were performed to individually position an array of four transponders from 2002 to 2005 . The resulting RMS variability for one day solutions, averaging the four transponder positions, ranged from $\pm 37-117 \mathrm{~mm}$ 
a) GPSA technique

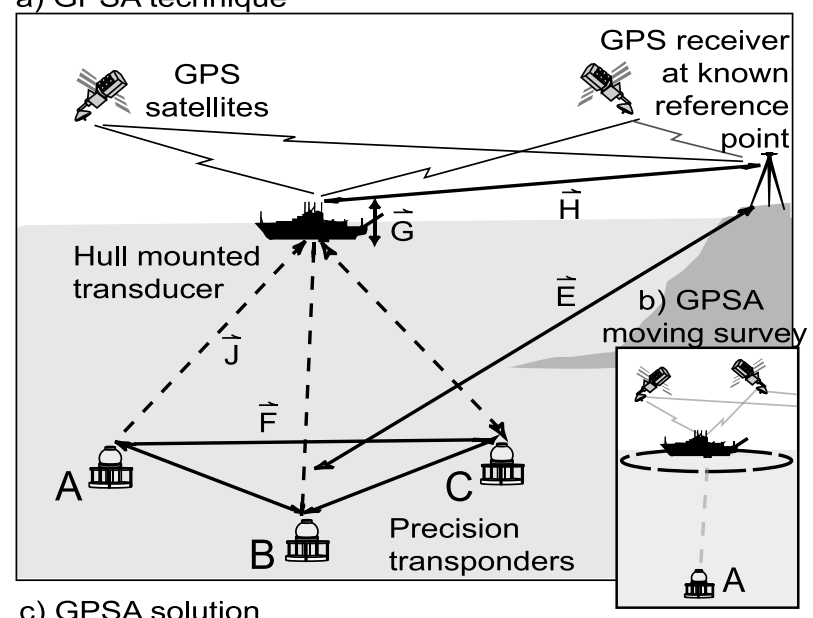

c) GPSA solution

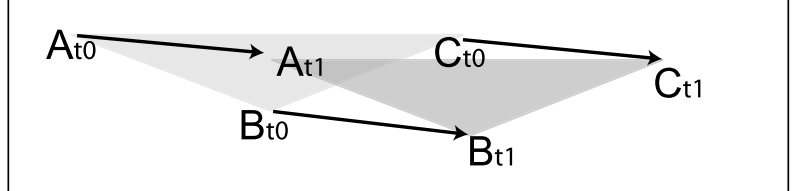

d) GPSA solution with transponder relocation

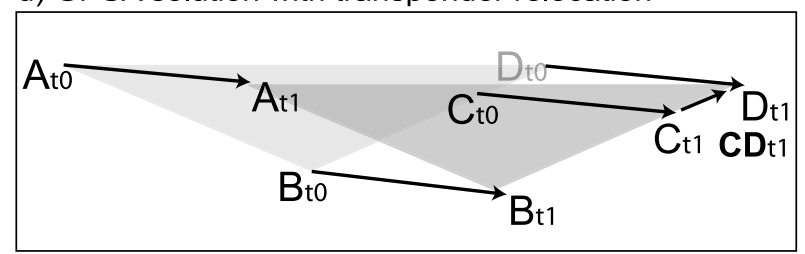

Fig. 1. a) The GPS-Acoustic approach to measure seafloor motion. Three precision transponders are deployed around the circumference of a circle with a radius equal to the nominal water depth. The transponders are spaced several kilometers apart and the relative positions $\vec{F}$ are measured by performing a moving GPSA survey (circle drive). Dual-frequency GPS carrier phase data sampled at $1 \mathrm{~Hz}$ at the ship and onshore provide the connection to sub-aerial reference stations $\vec{H}$. An optical survey connects shipboard GPS antenna phase centers to the acoustic hydrophone phase center $\vec{G}$. Two-way travel times of acoustic signals are collected between the ship and the transponder array $\vec{J}$. Vectors $\vec{F}, \vec{G}, \vec{H}$ and $\vec{J}$ combine to determine the horizontal components of $\vec{E}$. Maintaining the ship near the array center assures that acoustic velocity variations are primarily a function of depth and do not bias the horizontal components of $\vec{E}$. Survey times range from 80 to 120 hours in order to achieve sub-centimeter-level positioning. b) Single transponder circle drive around transponder A. c) Displacement of a rigid array of transponders $\mathrm{A}, \mathrm{B}$, and $\mathrm{C}$ from time $t_{0}$ to $t_{1}$. The position of each transponder is described by the latitude, $\phi$, longitude, $\lambda$ and height, $H$. d) Displacement of a rigid array of transponders A, B, and D from time $t_{0}$ to $t_{1}$ where transponder $\mathrm{D}$ is placed $1-3 \mathrm{~m}$ from acoustically inactive transponder $\mathrm{C}$ in time $t_{1}$. Position of transponder $\mathrm{D}$ at $t_{0}$ is unknown and estimated with the techniques discussed here to determine offset $\mathbf{C D}_{t_{1}}$.

east and $\pm 48-114 \mathrm{~mm}$ north with 48-192 hours of data (Fujita et al., 2006). Yamada et al., (2002) performed an error evaluation for single transponder positioning with uncertainties on the order of $180 \mathrm{~mm}$ using numerical simulations. Obana et al., (2000) has also used kinematic GPS and acoustic ranging to individually locate two transponders off the coast of Shirahama, southwest Japan and in Sagami Bay, central Japan. They estimated the positions of the individual seafloor transponders with 5 hours of data collection at each site. The standard error was estimated as $\pm 180 \mathrm{~mm}$ east and $\pm 140 \mathrm{~mm}$ north at the shallow site and $\pm 220 \mathrm{~mm}$ east and $\pm 150 \mathrm{~mm}$ north at the deep site.

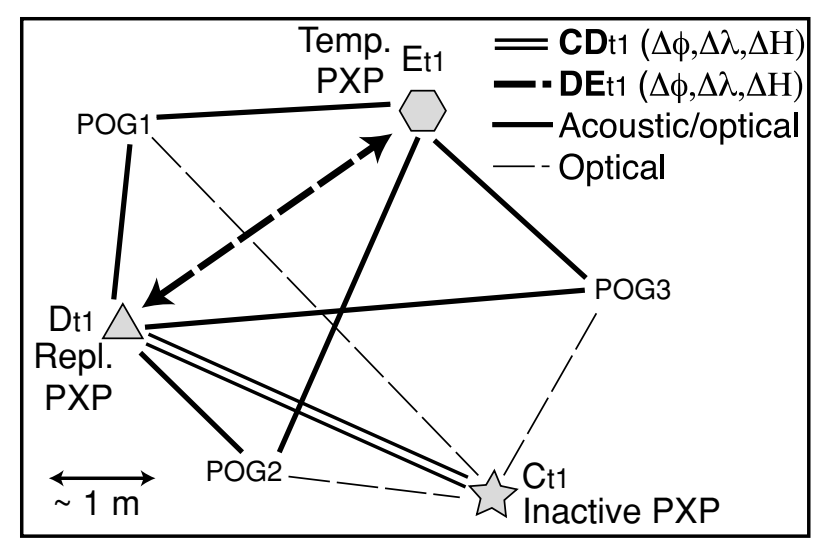

Fig. 2. Relocation network showing three POGO landings (e.g. POG1) and three transponders. The star represents the acoustically inactive transponder $\left(C t_{1}\right)$, the triangle represents the replacement transpon$\operatorname{der}\left(D t_{1}\right)$ and the hexagon represents the temporary transponder $\left(E t_{1}\right)$. Acoustic ranges are taken from the POGO transducer to the active transponders while optical ranges are taken from the POGO camera to each transponder. The latitude, longitude and height offsets from the replacement to the temporary transponder $\left(\mathbf{D E} t_{1}\right)$ are determined with the GPSA circle drive. The latitude, longitude and height offsets from the replacement to the inactive transponder $\left(\mathbf{C D} t_{1}\right)$ are determined with the acoustic/optical survey.

\section{GPS-Acoustic Technique}

The GPS-Acoustic (GPSA) technique, as operated by SIO, combines campaign-style kinematic GPS on a dynamically positioned vessel and acoustic travel time measurements from a ship to a rigid array of transponders. The transponder array includes three or four precision acoustic transponders on a circle with radius equal to the nominal water depth (Spiess et al., 1998). First, a moving GPSA survey is performed at each transponder (PXP) to determine the transponder's horizontal position with an uncertainty of $\pm 20 \mathrm{~cm}$ (Fig. 1(b)). The vertical position with this technique has an uncertainty of a meter. The vertical position is also independently determined by placing a seafloor survey instrument, equipped with a pressure sensor, near the transponder. Using the latitude, longitude, and depth of each transponder, an equi-angle point is determined such that the acoustic launch angle from the ship's hydrophone to each transponder is equal, creating uniformly spaced acoustic raypaths at the surface (Fig. 1(a)) (Spiess et al., 1998). Next, GPS, acoustic, and conductivity-temperature-depth (CTD) data are gathered for several days from near $(\sim 10 \mathrm{~m})$ the equi-angle position. Sound speed variability is highest in the top 200 meters and fluctuates at a higher frequency than can be measured with the CTD profiler. Maintaining position at the center of the array will ensure that each transponder's raypath is equally affected by the sound speed variability. This causes fluctuations in the vertical positioning of the array but not the horizontal.

GPS data are collected at $1 \mathrm{~Hz}$ from three tower-mounted antennas on the ship and are referenced to high-rate coastal GPS stations using a double differencing technique. The GPS data are processed using GIPSY/OASIS II software from NASA's Jet Propulsion Lab (Webb and Zumberge, 1997) and using techniques developed at SIO (Spiess et al., 1998; Chadwell and Bock, 2001), which provide the 


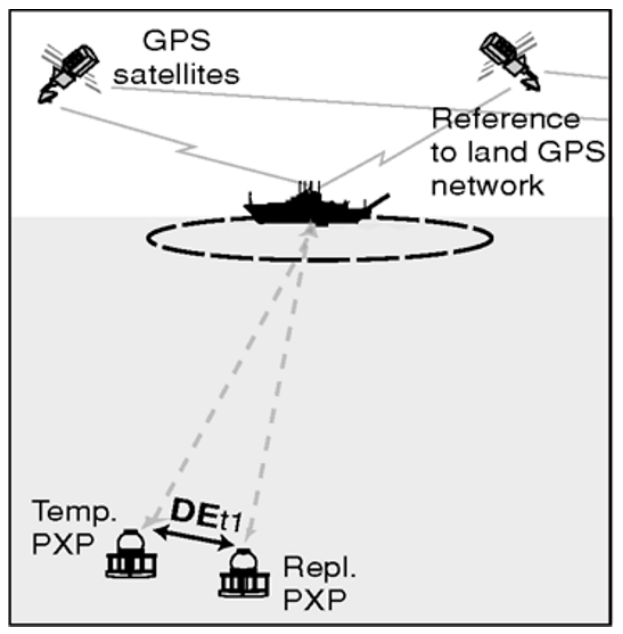

Fig. 3. Dual transponder GPSA circle drive around a temporary and replacement transponder to orient the observation network in latitude, longitude and height and estimate $\mathbf{D E} t_{1}(\sim 3 \mathrm{~m})$, the baseline of the two active transponders. The depth of the transponders is on the order of $\mathrm{km}$.

absolute position of the ships antennas with a repeatabillity of 10-20 $\mathrm{mm}$ in the horizontal (Miura et al., 2002), and 20-30 mm in the vertical (Chadwell and Bock, 2001). The position of the GPS antenna phase centers are transferred to the well-mounted hydrophone with a precision of 2$3 \mathrm{~mm}$ using a standard surveyors unit (Chadwell, 2003). This provides the GPS position of the hydrophone at each acoustic transmit and receive with a precision of 20-30 $\mathrm{mm}$. Sound speed is calculated from surface-to-seafloor CTD casts at the center of the array during GPSA operations and using equations from Chen and Millero, Del Grosso, and Wilson (Chen and Millero, 1977; Millero and Li, 1995; Del Grosso, 1974; Wilson, 1960). Sea surface height is estimated with both tide gauges and tide models to cover the time span of GPSA operations. The tidal contribution to sea surface height is used to shift the sound speed profile up or down in the acoustic raytrace solution (Spiess et al., 1998).

The GPS-Acoustic observations are combined in a leastsquares adjustment where the seafloor transponder's relative positions are held fixed and the entire array is allowed to move horizontally and vertically. In the following epoch, the GPSA survey is repeated and the adjustment, with the initial transponder positions at $t_{0}$, is performed again. Plate motion, $\mathbf{P}$, is represented by the average displacement in latitude and longitude of each transponder between times $t_{0}$ and $t_{1}$. Array displacement is shown in Fig. 1(c) where plate motion is represented by the vector

$$
\mathbf{P}=\frac{\mathbf{A}+\mathbf{B}+\mathbf{C}}{3},
$$

and

$$
\begin{aligned}
& \mathbf{A}=(\phi, \lambda, H)_{A t_{1}}-(\phi, \lambda, H)_{A t_{0}}, \\
& \mathbf{B}=(\phi, \lambda, H)_{B t_{1}}-(\phi, \lambda, H)_{B t_{0}}, \\
& \mathbf{C}=(\phi, \lambda, H)_{C t_{1}}-(\phi, \lambda, H)_{C t_{0}},
\end{aligned}
$$

such that $\mathbf{A}, \mathbf{B}$, and $\mathbf{C}$ are vectors of the displacement of transponders $\mathrm{A}, \mathrm{B}$ and $\mathrm{C}$, respectively. In the case where

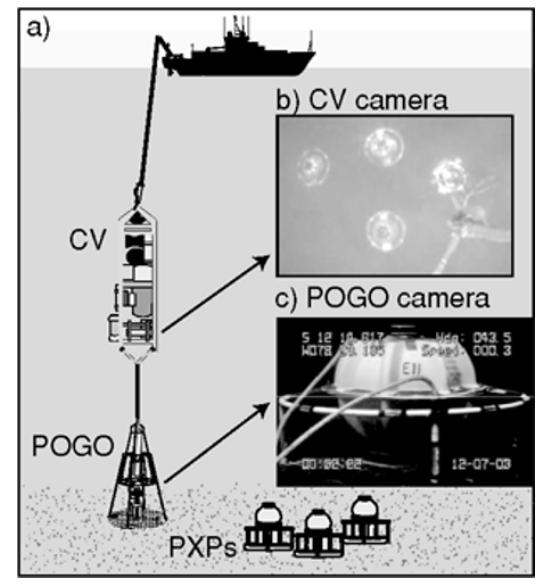

Fig. 4. a) Cartoon of the acoustic/optical survey with the ship, control vehicle (CV), POGO and PXPs. b) Digital image from the CV's down-looking camera illuminating the POGO, tethered below the CV, and the replacement, temporary and inactive transponders. c) Digital image from the POGO camera looking toward a transponder on the seafloor.

Table 1. Observables in the A/O survey for transponder depth.

\begin{tabular}{lc}
\multicolumn{1}{c}{ Observables } & $1-\sigma$ Uncertainty \\
\hline Camera pitch to PXP transducer $\left(\mu_{d c r}\right)$ & $\pm 0.4^{\circ}$ \\
POGO depth $\left(Z_{\text {pogo }}\right)$ & $\pm 3 \mathrm{~cm}$ \\
Travel time $(t t)$ & $\pm 3 \mu \mathrm{s}$ \\
Sound speed $(s s)$ & $\pm 0.015 \mathrm{~m} / \mathrm{s}$ \\
POGO X-plane tilt $\left(\eta_{x}\right)$ & $\pm 0.5^{\circ}$ \\
POGO Y-plane tilt $\left(\eta_{y}\right)$ & $\pm 0.5^{\circ}$ \\
Transducer height pixel count $\left(H_{d c r-p x l}\right)$ & \pm 2 pixels \\
Ring width pixel count $\left(W_{r n g-p x l}\right)$ & \pm 1 pixel \\
\hline
\end{tabular}

one transponder becomes inactive (PXP C in Fig. 1(d)), Eq. (1) becomes,

$$
\mathbf{P}=\frac{\mathbf{A}+\mathbf{B}+\mathbf{D}}{3},
$$

where

$$
\mathbf{D}=(\phi, \lambda, H)_{D t_{1}}-(\phi, \lambda, H)_{D t_{0}} .
$$

Given transponders $\mathrm{C}$ and $\mathrm{D}$ are separated by $3 \mathrm{~m}$, their tectonic displacement is assumed to be equal, i.e., $\mathbf{C}=\mathbf{D}$, which after substituting Eqs. (4) and (6) becomes,

$$
\begin{aligned}
(\phi, \lambda, H)_{D t_{0}}= & (\phi, \lambda, H)_{C t_{0}} \\
& +\left[(\phi, \lambda, H)_{D t_{1}}-(\phi, \lambda, H)_{C t_{1}}\right],
\end{aligned}
$$

where $(\phi, \lambda, H)_{D t_{0}}$ would be the position of the replacement transponder if it had existed in the previous epoch. This is required to initialize the $t_{1}$ GPSA least-squares solution. The value $(\phi, \lambda, H)_{C t_{0}}$ is determined from the circle drive in the epoch $t_{0}$. The term $\left[(\phi, \lambda, H)_{D t_{1}}-(\phi, \lambda, H)_{C t_{1}}\right]$ represents the offset from the replacement to the inactive transponder and can be more simply written as

$$
\mathbf{C D}_{t_{1}}=(\phi, \lambda, H)_{D t_{1}}-(\phi, \lambda, H)_{C t_{1}} .
$$

$\mathbf{C D}_{t_{1}}$ is determined as offsets in latitude, longitude and height by a GPSA circle drive with range differencing and an acoustic/optical survey at the seafloor. These two techniques are detailed in the following sections. 

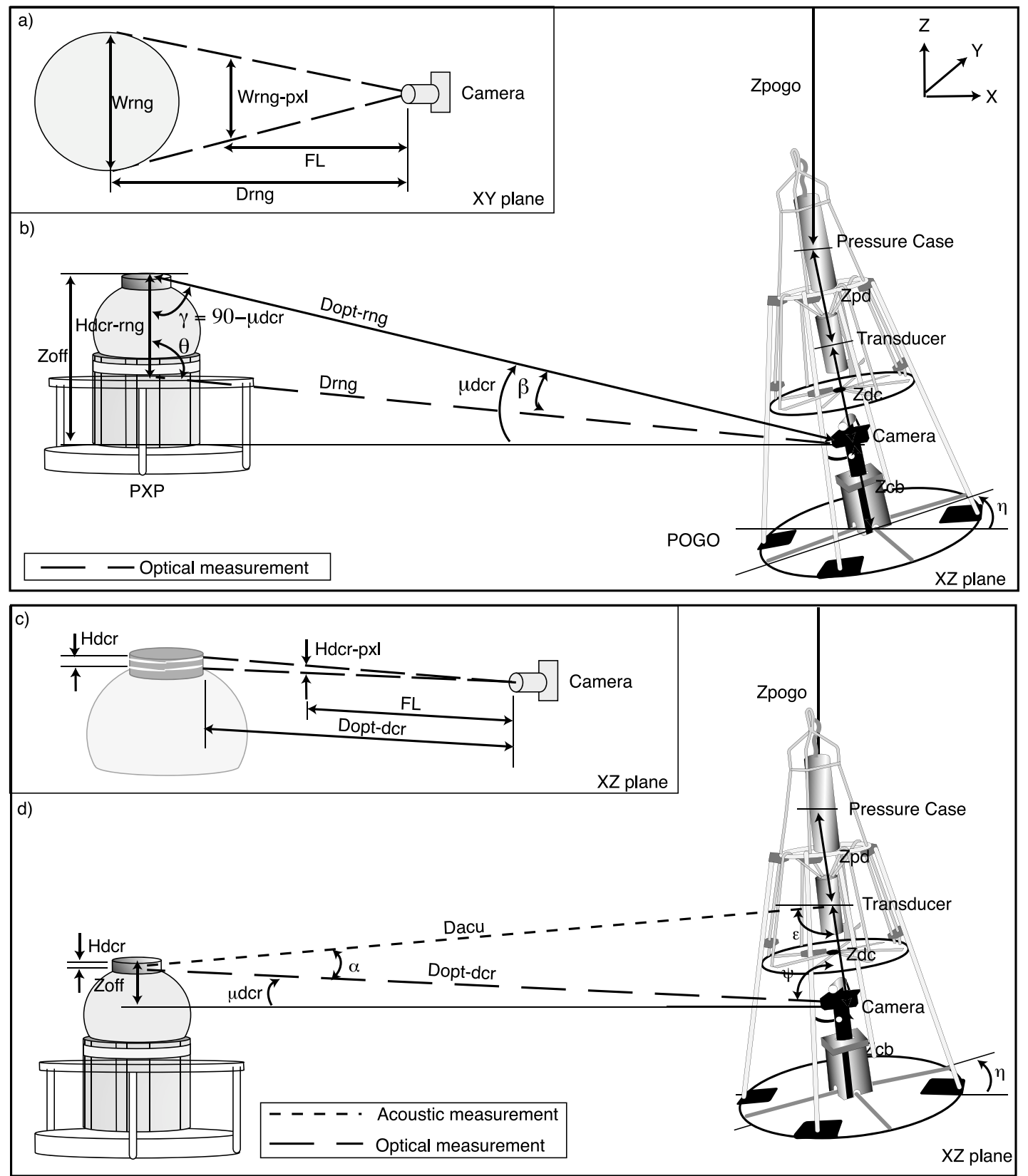

Fig. 5. a) Relating the optical range from the POGO camera to the ring, $D_{r n g}$, and the focal length of the camera, $F L$. b) Measuring the depth of the PXP transducer from sighting the PXP metal frame (ring). c) Relating the optical range from the POGO camera to the transducer, $D_{o p t-d c r}$, and the focal length of the camera, $F L$. d) Measuring the depth of the PXP transducer from sighting the PXP transducer or using the acoustic range.

\section{Range Differencing}

A temporary, recallable transponder (PXP E) is placed in the vicinity $(\sim 3 \mathrm{~m})$ of the inactive and replacement transponders, shown in the relocation network in Fig. 2. The active transponders are used to constrain the geodetic azimuth and scale of the network. The ship drives a 1-nm-radius circle centered on the active transponders while acoustically interrogating (Fig. 3). First, we calculate the absolute position of the replacement transponder $\left[(\phi, \lambda, H)_{D t_{1}}\right]$ with the GPS-Acoustic circle drive technique discussed in Section 3. Next, we calculate the latitude, longitude and height offsets $\left(\mathbf{D E}_{t_{1}}\right.$ or bold, dashed line in Fig. 2) by differencing the travel times to the replacement and temporary transponders at each epoch. The proximity of the active transponders $(1-3 \mathrm{~m})$ creates a common acous- tic raypath, eliminating any unmodeled variability in the sound speed and providing the baseline with millimeter resolution. The geodetic azimuth of the baseline components is controlled by GPS and the uncertainty is constrained by

$$
\frac{\sigma_{D E}}{\mathbf{D E}_{\mathbf{t}_{1}}} \propto \frac{\sigma_{G P S}}{\phi_{\text {circle }}},
$$

where $\sigma_{G P S}$ represents the GPS positional uncertainty and $\phi_{\text {circle }}$ is the diameter of the circle drive. The ratio of the baseline to the circle diameter is roughly $1: 1800$, reducing the baseline uncertainty, $\sigma_{D E}$, by a factor of $0.05 \%$.

\section{Acoustic/Optical Survey}

An underwater surveying instrument is used to acoustically interrogate the active transponders (solid lines in 
Fig. 2) and optically sight each transponder (gray, dashed lines in Fig. 2). These data are used in a least-squares adjustment to determine the latitude, longitude and height offsets from the replacement to the inactive transponder $\left(\mathbf{C D} t_{1}\right.$ or bold, double line in Fig. 2) in the global reference frame. The acoustic and optical (A/O) survey is performed with the Marine Physical Lab Control Vehicle (CV) and a surveying instrument, POGO, shown in Fig. 4. The CV is equipped with lateral thrusters, a pressure gauge, bottom looking hydrophone and a down looking camera (Fig. 4(b)) while the POGO carries 4 Paroscientific Digiquartz pressure gauges, a temperature sensor, conductivity sensor, tilt-meter, a sidefacing transducer and a camera with surface controlled pan and tilt (Fig. 4(c)). After sufficient temperature sensor equilibration within 100 meters of the seafloor, the POGO package is placed on the seafloor at three sites within 2 meters of the transponders such that each PXP is clearly visible. The pan and tilt video camera captures painted scale lines on the PXP transducer and opposite edges of the 1-m-diameter metal ring encompassing the PXP. Optical ranges are calculated from the stored digital image by counting pixels of predetermined lengths on the PXP frame. The POGO transducer interrogates the two active transponders, once every 10 seconds, providing 10-20 travel times during each, roughly 2 minute survey. The range is calculated using the sound speed profile discussed above, extrapolated to the depth of the transponders. The POGO pressure record is averaged between the 4 pressure gauges and corrected for the ocean surface tidal signal, water column density, and atmospheric pressure. The remainder of this section presents the solution for transponder depth, followed by the solution for the network baselines.

\subsection{Transponder depth}

The PXP consists of a circular metal frame around a pressure-resistant glass sphere housing the electronics and a cylindrical transducer at the top. The depth of the PXP transducer is calculated from the depth of the POGO pressure case, $Z_{\text {pogo }}$, plus or minus a depth offset, $Z_{\text {off }}$, from the POGO camera to the PXP transducer (Fig. 5). Calculating $Z_{\text {off }}$ requires measurement of the slant distance to the PXP. There are three methods to determine the slant distance; optical sighting to either the transducer or frame-ring and acoustic ranging to the active PXP transducer. The preferred optical target is the ring, which is $\sim 1 \mathrm{~m}$ wide and is more precise than sighting the transducer, $\sim 0.025 \mathrm{~m}$ tall. The latter is only used if the ring sighting is obscured. The observables and uncertainties collected during the survey are shown in Table 1. For the optical measurement of the ring (Fig. 5a) the law of similar triangles allows the range from the camera to the opposite edges of the ring to be written as

$$
D_{r n g}=\frac{F L * W_{r n g}}{W_{r n g-p x l}},
$$

where $W_{r n g}$ was measured prior to deployment, $W_{r n g-p x l}$ is from the digital image and $F L$ is the camera's focal length, determined by self-calibration. From Fig. 5(b), let $\gamma$ be defined as

$$
\gamma=90^{\circ}-\mu_{d c r}
$$

where $\mu_{d c r}$ is the pitch of the camera measured with an onboard tilt sensor. Using the law of sines in the triangle with angles $\theta, \beta, \gamma$,

$$
\beta=\arcsin \left[\frac{H_{d c r-r n g} \sin \gamma}{D_{r n g}}\right]
$$

and the range to the PXP transducer is

$$
D_{o p t-r n g}=\frac{D_{r n g} \sin \theta}{\sin \gamma},
$$

where

$$
\theta=180^{\circ}-\beta-\gamma .
$$

The depth offset from the camera to the transducer is

$$
Z_{o f f}=D_{o p t-r n g} \sin \mu_{d c r} .
$$

The final depth of the PXP transducer from the ring sighting, incorporating the tilt of the POGO frame, $\eta$, is

$$
Z_{\text {pxp }}^{\text {opt-rng }}=Z_{\text {pogo }}+\left(z_{p d}+z_{d c}\right) \cos \eta-Z_{o f f},
$$

where the height from the midpoint of the POGO pressure case to the POGO transducer, $Z_{p d}$, and the height from the POGO transducer to the camera, $Z_{d c}$, are measured prior to deployment.

For the optical measurement of the transducer (Fig. 5(c)) the relationship between the transducer height, $H_{d c r}$, and the focal length of the camera is

$$
D_{o p t-d c r}=\frac{F L * H_{d c r}}{H_{d c r-p x l}},
$$

where $H_{d c r}$ was measured prior to deployment and $H_{d c r-p x l}$ is from the digital image. The height between the PXP transducer and POGO camera from Fig. 5(d) is

$$
Z_{o f f}=D_{o p t-d c r} \sin \mu_{d c r},
$$

and the final depth of the PXP transducer using the transducer sighting is

$$
Z_{p x p}^{o p t-d c r}=Z_{\text {pogo }}+\left(z_{p d}+z_{d c}\right) \cos \eta-Z_{o f f} .
$$

The depth of active transponders can be determined using the acoustic range, $D_{a c u}$, shown in Fig. 5(d). The acousticbased depth calculation is more accurate than the optical due to the higher uncertainty in counting pixels. The range of the acoustic signal is,

$$
D_{a c u}=t t \frac{s s}{2},
$$

where $t t$ is the two-way or round trip travel time from the POGO to the PXP transducer and $s s$ is the sound speed. Let $\psi$ be defined as

$$
\psi=90^{\circ}-\mu_{d c r}-\eta
$$

Using the law of sines in the triangle with angles $\alpha, \epsilon, \psi$,

$$
\alpha=\arcsin \left(z_{d c} \frac{\sin \psi}{D_{a c u}}\right),
$$


Table 2. Observables and unknown parameters in the A/O least-squares adjustment.

\begin{tabular}{ll}
\hline \multicolumn{1}{c}{ Observables and 1- $\sigma$ Uncertainty } & \multicolumn{1}{c}{ Parameters } \\
\hline Camera Pitch $\left(\mu_{d c r}\right) \pm 0.4^{\circ}$ & Focal length \\
POGO depth $\left(Z_{\text {pogo }}\right) \pm 3 \mathrm{~cm}$ & Optical distance bias \\
Travel time $(t t) \pm 3 \mu \mathrm{s}$ & Vertical angle bias \\
Sound speed $(s s) \pm 0.015 \mathrm{~m} / \mathrm{s}$ & Temp. PXP time bias \\
Distance to the rng $\left(d_{r n g}\right)$ & Repl. PXP time bias \\
Baseline DE $t_{1} \pm 2.3 \mathrm{~mm}$ & $\phi, \lambda, H$ Repl. PXP at $t_{1}$ \\
Initial position $(\phi, \lambda, H)_{C t_{0}} \pm 200 \mathrm{~mm}$ & $\phi, \lambda, H$ Temp. PXP at $t_{1}$ \\
Transducer height pixel count $\left(H_{d c r-p x l}\right) \pm 2$ pixels & $\phi, \lambda, H$ Each POGO landing at $t_{1}$ \\
Ring width pixel count $\left(W_{r n g-p x l}\right) \pm 1$ pixel & \\
\hline
\end{tabular}

Table 3. 1- $\sigma$ east, north, and up uncertainties $( \pm \mathrm{mm})$ of $\mathbf{D E}_{t_{1}}$ from the dual PXP GPSA circle drive.

\begin{tabular}{cccc}
\hline Array [TEMP.-REPL. PXP] & $\sigma_{e}$ & $\sigma_{n}$ & $\sigma_{u}$ \\
\hline Cascadia [E4-E5] & 1.8 & 1.6 & 0.7 \\
Cleft [E4-E6] & 1.1 & 1.0 & 0.5 \\
Peru [E4-E11] & 1.7 & 2.1 & 0.8 \\
Average & 1.6 & 1.6 & 0.7 \\
\hline
\end{tabular}

Table 4. Details of the A/O survey least-squares adjustments.

\begin{tabular}{cccc}
\hline & Cascadia & Cleft & Peru \\
\hline POGO landings & 6 & 3 & 6 \\
No. observations & 47 & 29 & 50 \\
No. unknowns & 32 & 23 & 32 \\
No. iterations & 8 & 5 & 5 \\
$d . f$. & 19 & 10 & 22 \\
$c$ & 1.75 & 1.79 & 1.71 \\
$\hat{\sigma}_{0}^{2}($ Eqn 39) & 0.31 & 0.62 & 0.31 \\
\hline
\end{tabular}

and

$$
D_{o p t-d c r}=\frac{\sin \epsilon}{\sin \psi} D_{a c u},
$$

where

$$
\epsilon=180^{\circ}-\alpha-\psi .
$$

The depth offset from the POGO camera to the PXP transducer is

$$
Z_{o f f}=D_{o p t-d c r} \sin \mu_{d c r},
$$

and the final PXP depth using the acoustic signal is

$$
Z_{p x p}^{a c u}=Z_{\text {pogo }}+\left(z_{p d}+z_{d c}\right) \cos \eta-Z_{o f f} .
$$

\subsection{Replacement-Inactive transponder baseline}

A linearized least-squares adjustment with the above observation models is used to determine the position of all POGO landings and active/inactive transponders. The overdetermined system of observations, $\mathbf{O}$, and unknown parameters, $\mathbf{x}$, are listed in Table 2 . They are related by

$$
\begin{array}{r}
\mathbf{O}=f(\mathbf{x}), \\
\mathbf{c}=g(\mathbf{x}),
\end{array}
$$

where $f(\mathbf{x})$ are the functional relationships given in Section 5.1 and $g(\mathbf{x})$ are the inner constraints. Inner constraints

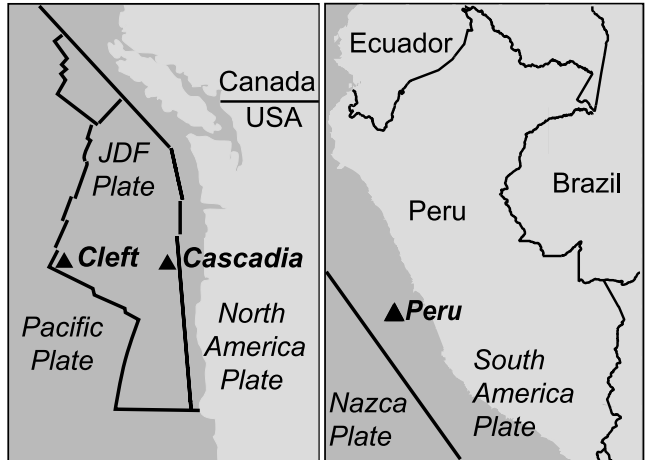

Fig. 6. Transponder arrays (black triangles) requiring a replacement (Left) $25 \mathrm{~km}$ east of the Cleft segment of the Juan de Fuca ridge $\left(44.5^{\circ} \mathrm{N}\right), \sim 60$ $\mathrm{km}$ west of the south Cascadia subduction zone, and (Right) $50 \mathrm{~km}$ east of Peru-Chile trench $\left(12^{\circ} \mathrm{S}\right)$.

fix the net horizontal translation and azimuthal rotation of the network to zero (Leick, 2004). The remaining coordinate frame definitions, vertical translations, tilting of the horizontal plane and scale are controlled by the observations. The linear model is defined by

$$
\begin{aligned}
& \mathbf{v}_{o}=\mathbf{A x}+\mathbf{L}, \\
& \mathbf{v}_{c}=\mathbf{G x}+\mathbf{C},
\end{aligned}
$$

where $\mathbf{v}_{o}$ defines the residuals of the observations, $\mathbf{v}_{c}$ defines the residuals of the constraints, $\mathbf{A}=\delta f / \delta \mathbf{x}$ and $\mathbf{G}=\delta g / \delta \mathbf{x}$, evaluated at $x$. For the inner constraint solution, $\mathbf{C}=\mathbf{0}$ and the covariance matrix of the constraints, $\Sigma_{c}=\mathbf{0}$. The non-unique matrix $\mathbf{E}^{T}$ (Leick, 2004) is introduced to form the null space of $\mathbf{A}$ such that

$$
\mathbf{A} \mathbf{E}^{T}=\mathbf{0} .
$$

The corrections to the initial estimate of unknowns, $\mathbf{x}_{o}$, can be written as

$$
\Delta \mathbf{x}=-\mathbf{Q}_{\Delta \mathbf{X}} \mathbf{A}^{T} \Sigma_{0}^{-1} \mathbf{L},
$$

where

$$
\mathbf{Q}_{\Delta \mathbf{X}}=\left(\mathbf{A}^{T} \Sigma_{0}^{-1} \mathbf{A}+\mathbf{E}^{T} \mathbf{E}\right)^{-1}-\mathbf{E}^{T}\left(\mathbf{E} \mathbf{E}^{T} \mathbf{E} \mathbf{E}^{T}\right)^{-1} \mathbf{E}
$$

and the covariance matrix of the adjusted parameters is

$$
\Sigma_{x}=\sigma_{0}^{2} \mathbf{Q}_{\Delta \mathbf{X}} .
$$



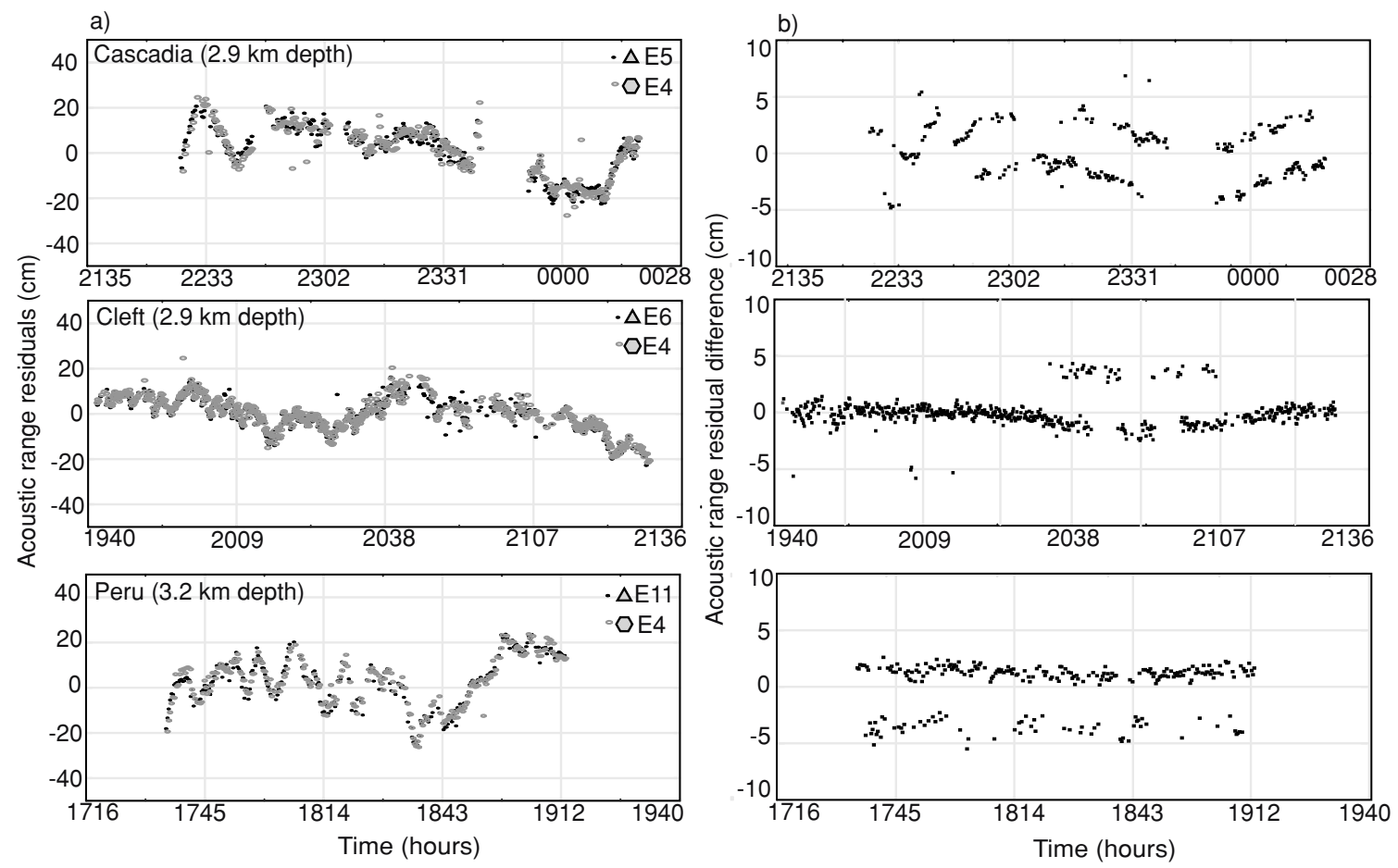

Fig. 7. a) Acoustic range residuals from a circle drive at Cascadia. Temporary transponder (grey ellipse) is always E4, replacement transponder (black circle) E5; Cleft array with replacement transponder E6; Peru array with replacement transponder E11. b) Difference between replacement and temporary transponder range residuals for each survey.

The new estimate of the unknown parameters can be written

$$
\hat{\mathbf{x}}=\mathbf{x}_{o}+\Delta \mathbf{x}
$$

The adjustment is reiterated until $\Delta \mathbf{x}$ is below a threshold, in this case $0.001 \mathrm{~m}$. The observations are analyzed for outliers with Pope's method based on the Studentized residual, $\tau$, and rejected if greater than a critical value, $c$, based on a risk level of $\alpha=5 \%$, or 95\% confidence (Caspary, 1988; Leick, 2004).

A redundancy number $\left(r_{i}\right)$ is calculated to investigate each observation's contribution to the redundancy of the model, i.e., the degrees of freedom. This value is defined by

$$
r_{i}=q_{i} p_{i}\left(0<r_{i}>1\right)
$$

where $q_{i}$ is the diagonal element of the cofactor matrix for the residuals $\left(\mathbf{Q}_{v}\right)$ (Leick, 2004) and $p_{i}$ is the weight of the $i^{\text {th }}$ observation. The redundancy number exposes weak parts of the adjustment whereby if $r_{i}$ nears 0 , the observation is uniquely used in the solution and it does not increase the degrees of freedom $\left(\Sigma r_{i}=d . f\right.$.). If $r_{i}$ equals 1 , the observation is redundant and increases the $d$.f. by 1 . Observations with a $r_{i}<0.3$ should be avoided to ensure the model can identify when the observation is an outlier (Caspary, 1988). That threshold, i.e. each observation's maximum error that can be detected as an outlier is the marginally detectable blunder (mdb), $\nabla_{i}$, and is defined as

$$
\nabla_{i}=\frac{u_{\alpha, \beta}}{\sqrt{r_{i}}} \sigma_{i}
$$

where $u_{\alpha, \beta}$ represents risk level, $\alpha=5 \%$, and probability of type II error, $\beta=20 \%$ (Caspary, 1988) and $\sigma_{i}$ is observation uncertainty. A high mdb indicates an unreliable part of the network where the geometry or set of observations should be strengthened. The effect of each observation's mdb on the unknowns is

$$
\nabla x_{i}=\left(\mathbf{A}^{T} \Sigma_{0}^{-1} \mathbf{A}\right)^{-1} \mathbf{A}^{T} \Sigma_{0}^{-1} \nabla_{i}
$$

which represent coordinate shifts to the POGO and PXP positions. The a priori variance of unit weight,

$$
\hat{\sigma}_{0}^{2}=\frac{\hat{\mathbf{v}}^{T} \Sigma_{0}^{-1} \hat{\mathbf{v}}}{d . f .}
$$

where $\hat{\mathbf{v}}$ is the residual matrix, is also sensitive to the mdbs. This value should converge to 1 if the model is consistent with the observations and their uncertainties.

\section{Results: GPS-Acoustic Circle Drive}

Two relocations were performed in arrays on the oceanic Juan de Fuca plate, one at the south Cascadia subduction zone, and one east of the Juan de Fuca spreading ridge at the Cleft segment. A third relocation was performed in an array on the submerged continental South America plate offshore Peru (Fig. 6). The GPSA circle drive at each site provided the offset in east, north, and up between the temporary and replacement transponders, $\mathbf{D E}_{t_{1}}$. Fig. 7(a) shows the acoustic range residuals of the GPS-Acoustic circle drives in the Cascadia, Cleft and Peru arrays. The range residuals show roughly $40 \mathrm{~cm}$ peak to peak variation due to the changing sound speed structure in the upper water column that is common to both ranges. Differencing the acoustic range residuals of the replacement and temporary transponder is a measure of the repeatability of $\mathbf{D E} \mathbf{t}_{t_{1}}$ (Fig. 7(b)). The average residual difference is less than $1 \mathrm{~cm}$ at each site though 
Table 5. Adjusted output focal length and biases.

\begin{tabular}{cccc}
\hline Parameter & Cascadia & Cleft & Peru \\
\hline Focal length dcr $(\mathrm{m})$ & $1528.562 \pm 46.730$ & $1800.150 \pm 66.975$ & $1697.240 \pm 49.810$ \\
Opt. dist. bias (E-5 units) & $-7.23 \pm 4.11$ & $-3.31 \pm 5.32$ & $5.25 \pm 4.21$ \\
Vert. angle bias (dec. deg) & $-2.14 \mathrm{E}-13 \pm 6.75 \mathrm{E}-9$ & $-4.81 \mathrm{E}-14 \pm 7.87 \mathrm{E}-9$ & $-2.51 \mathrm{E}-14 \pm 5.55 \mathrm{E}-9$ \\
Temp. PXP bias (sec) & $-9.36 \mathrm{E}-15 \pm 6.75 \mathrm{E}-9$ & $2.86 \mathrm{E}-15 \pm 7.87 \mathrm{E}-9$ & $1.43 \mathrm{E}-14 \pm 5.55 \mathrm{E}-9$ \\
Repl. PXP bias (sec) & $-2.24 \mathrm{E}-14 \pm 6.75 \mathrm{E}-9$ & $-2.09 \mathrm{E}-15 \pm 7.87 \mathrm{E}-9$ & $-1.38 \mathrm{E}-14 \pm 5.55 \mathrm{E}-9$ \\
\hline
\end{tabular}

Table 6. 1- $\sigma$ east, north, and up uncertainties $( \pm \mathrm{mm})$ and correlation coefficients $(\rho)$ of $\mathbf{C D}_{t_{1}}$ from the A/O survey.

\begin{tabular}{ccccccc}
\hline Array [INACTIVE-REPL. PXP] & $\sigma_{e}$ & $\sigma_{n}$ & $\sigma_{u}$ & $\rho_{e, n}$ & $\rho_{e, u}$ & $\rho_{n, u}$ \\
\hline Cascadia [C3-E5] & 51 & 53 & 20 & -0.61 & -0.02 & 0.02 \\
Cleft [C7-E6] & 51 & 39 & 23 & 0.73 & 0.01 & 0.01 \\
Peru [D17-E11] & 33 & 42 & 17 & 0.45 & -0.01 & -0.02 \\
RMS & 45 & 45 & 20 & 0.59 & 0.02 & 0.02 \\
\hline
\end{tabular}

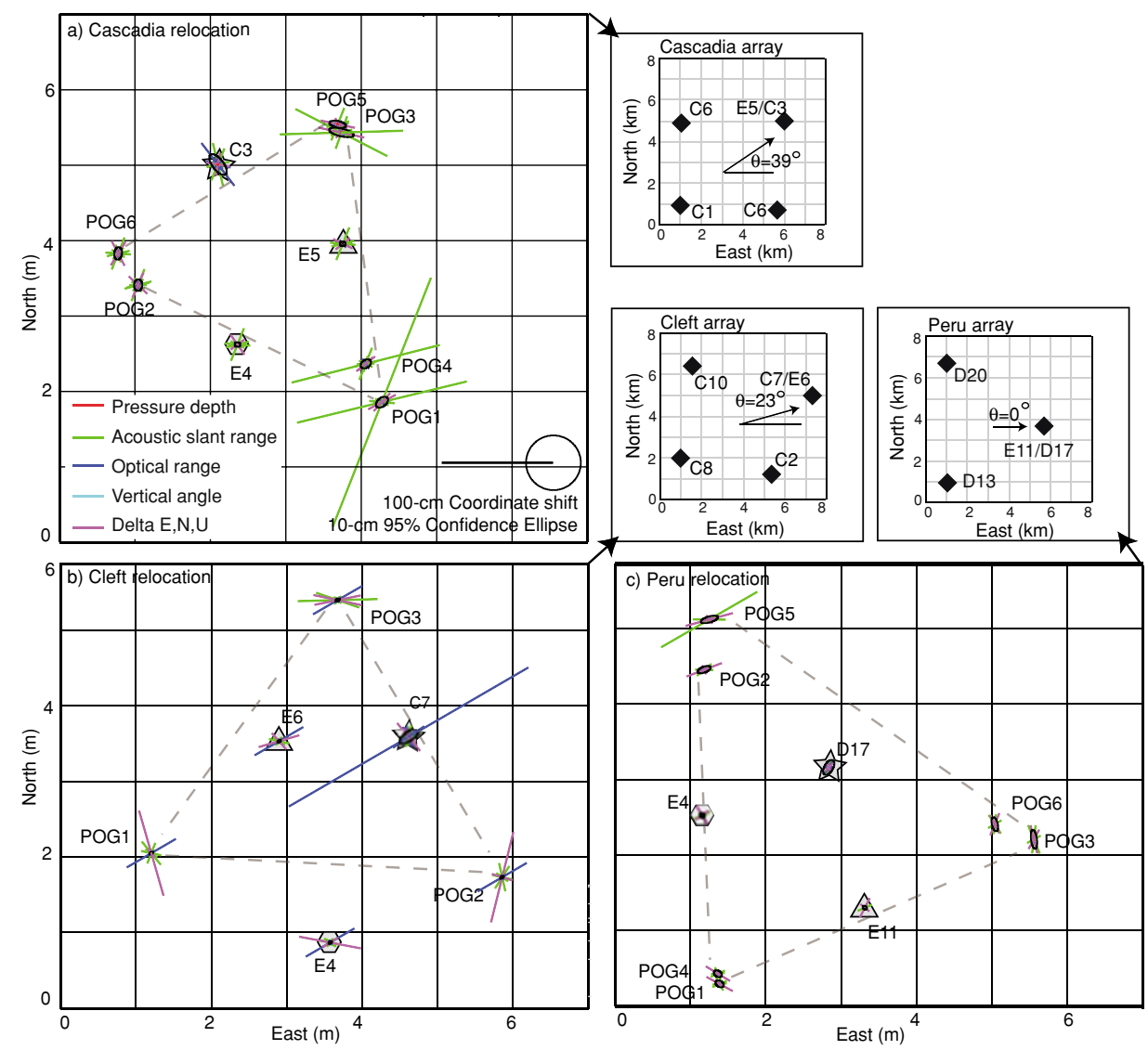

Fig. 8. a) Plot of the $95 \%$ confidence-level positional uncertainty (black ellipse) from Cascadia array least-squares adjustment for each POGO landing (e.g., POG1), active (E5, E4), and inactive transponders (C3). Straight colored lines represent potential coordinate shifts due to marginally detectable blunders in each observation. Dashed gray lines show the area inscribed by the POGO landings. b) Cleft array least-squares adjustment for each POGO landing, active (E6, E4), and inactive transponders (C7). c) Peru array least-squares adjustment for each POGO landing, active (E11, E4), and inactive transponders (D17). Arrows point to greater array orientation.

peak jumping during signal correlation can cause a $5 \mathrm{~cm}$ offset. The positional uncertainty of the active-temporary transponder baselines are shown in the Table 3.

\section{Results: A/O Survey}

Six POGO landings were performed around the Cascadia and Peru relocation sites and three around the Cleft. Two landings are the minimum required to have an exact solution, while three or more produces an overdetermined solution. Table 4 provides a description of each A/O survey including the number of observations and unknowns after outliers have been removed. As mentioned in Section 5.2, the a posteriori variance of unit weight should converge to 1 when the observation uncertainties and model are consistent. The generally low values $(<1)$ suggest that some assumed observation uncertainties provided as model input 
Table 7. Number of transponders per array $(N)$ and radial and tangential $( \pm \mathrm{mm})$ contribution to array position uncertainty.

\begin{tabular}{ccccc}
\hline Array & $N$ & $\theta^{\circ}$ & $\frac{\sigma_{\text {rad }}}{\sqrt{N}}$ & $\frac{\sigma_{\tan }}{\sqrt{N}}$ \\
\hline Cascadia & 4 & 39 & 16 & 32 \\
Cleft & 4 & 23 & 29 & 13 \\
Peru & 3 & 0 & 19 & 24 \\
\hline
\end{tabular}

have been over-estimated. Table 5 shows the adjusted focal length and optical bias as well as 1- $\sigma$ uncertainty. Three additional biases were included to allow the solution to selfcalibrate. These are a vertical angle bias and two timing biases, one for each active transponder. Inspection of Table 5 shows that these biases have a negligible effect on the solution.

The average uncertainty of the replacement transponder position relative to the inactive, $\mathbf{C D}_{t_{1}}$, shown in Table 6 , is $\pm 45 \mathrm{~mm}$ east and north. Figure 8 show the adjusted POGO and PXP positions, 95\% confidence ellipses and mdb coordinate shifts at Cascadia, Cleft, and Peru. At Cascadia, two large potential coordinate shifts due to acoustic slant range mdb's trend nearly parallel to the POG5-POG6 baseline. The solution is not affected by removing these observations, rather they indicate that the survey geometry is poor and that the acoustic ranges could shift the POGO positions if not constrained by other observations. At the Cleft array, the POGO landing sites do not inscribe PXP E4. The survey geometry produces one large optical range blunder to potentially shift C7's estimated position perpendicular to the POG2-POG3 and temporary-replacement PXP baselines. Again, this observation does not affect the final solution. The survey geometry in Peru produced a well constrained solution with small coordinate shifts and the lowest uncertainty of $\mathbf{C D}_{t_{1}}$.

The uncertainty of the array position is a combination of the positional uncertainty of each transponder in the array. The uncertainty added by performing a relocation is dependent on the number of transponders in the array $(N)$, their relative location, and the fit of the A/O survey observations in the least-squares adjustment. Collecting acoustic data from the center of the array makes the GPSA technique most sensitive to positional shifts in the radial direction and insensitive to shifts in the tangential direction. The east and north uncertainty of $\mathbf{C D}_{t_{1}}$ is rotated using

$$
\Sigma_{r, \theta}=\mathbf{G} \Sigma_{e, n} \mathbf{G}^{T},
$$

where the rotation matrix is defined by

$$
\mathbf{G}=\left[\begin{array}{cc}
\cos \theta & \sin \theta \\
-\sin \theta & \cos \theta
\end{array}\right] .
$$

Table 7 shows each relocation's radial and tangential contribution to array position uncertainty, where the angle $\theta$ is indicated for each array in Fig. 8. The range of uncertainty $\left(\sigma_{\text {rad }} / \sqrt{N}\right)$ added by the relocations discussed here is $\pm 16-$ $29 \mathrm{~mm}$.

\section{Simulation}

The geometry of the POGO landings around the transponders influences the uncertainty of $\mathbf{C D}_{t_{1}}$. Since

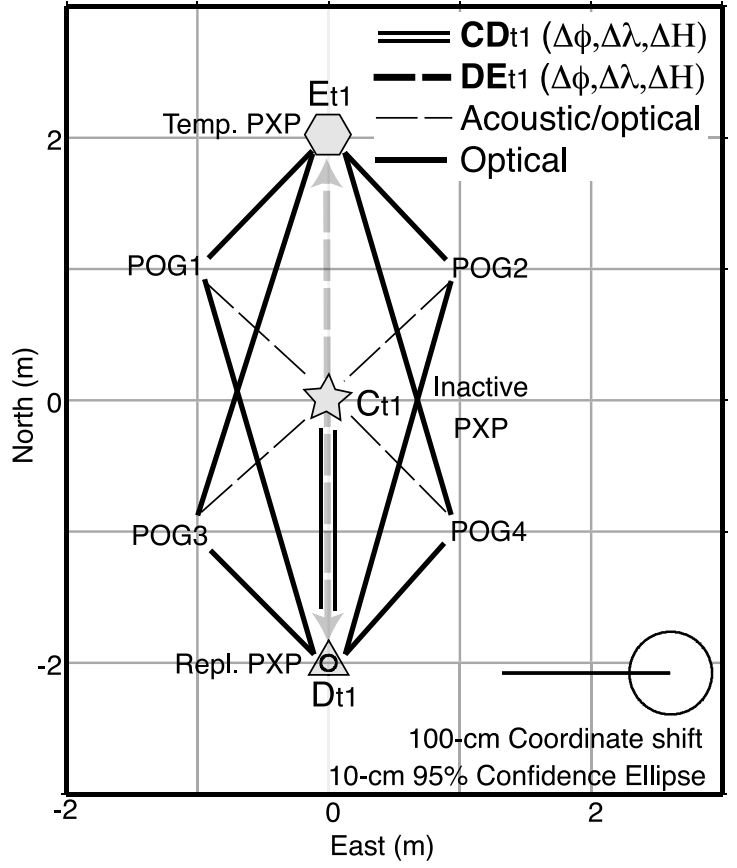

Fig. 9. Network simulation to better constrain the uncertainty of replacement-inactive transponder baseline, $\mathbf{C D}_{t_{1}}$.

the optical measurements have the highest uncertainties, 4 POGO landings as shown in Fig. 9, would better constrain the POGO positions relative to the inactive PXP. The temporary and replacement transponders should be placed on either side of the inactive transponder to provide both the orientation of the network and a scale measurement that constrains $\mathbf{C D}_{t_{1}}$ inside of $\mathbf{D E}_{t_{1}}$. A simulation was performed using this geometry and varying the A/O survey input within the standard deviation of each observation. The uncertainty of $\mathbf{C D}_{t_{1}}$ was roughly $\pm 15 \mathrm{~mm}$ east and north. Assuming an array of 4 transponders where $\theta=45^{\circ}$, the uncertainty added to the array position is $\pm 10 \mathrm{~mm}$.

\section{Conclusions}

The GPS-Acoustic technique can be used to accurately measure horizontal plate motion on the seafloor. Inevitably, failing transponders will have to be replaced as this technique continues to evolve. Three successful examples were discussed from the Cleft segment of the Juan de Fuca Ridge, the south Cascadia subduction zone and on the South America plate. A dual-transponder circle drive provides the baseline, including geodetic azimuth, between two active transponders with an average uncertainty of $\pm 1.6 \mathrm{~mm}$ east and north. The acoustic/optical survey is used to measure transponder depth with an uncertainty of $\pm 20 \mathrm{~mm}$ and the replacement-inactive PXP baseline with an average uncertainty of $\pm 45 \mathrm{~mm}$ east and north. The baseline is used in Eq. (7) to calculate $(\phi, \lambda, H)_{D t_{0}}$, which is used in the GPSA solution as the a priori position for the replacement transponder. These techniques are necessary to continue referencing previous epochs of GPSA data from a transponder array. The uncertainty of the $t_{1}$ array position is increased by the relocation, adding between $\pm 16-29 \mathrm{~mm}$. The relatively high uncertainty of optical measurements is the limiting fac- 
tor of this technique. However it's effect can be reduced and the method improved by strengthening the survey geometry in any future relocations. The optimal network would have replacement and temporary transponders on either side of the inactive transponder and at least two POGO landings on either side. Simulations show the uncertainty added to the array position decreases to less than $\pm 10 \mathrm{~mm}$.

Acknowledgments. Concept conceived by Dave Chadwell, John Hildebrand, and Fred Spiess. Katie Phillips and Neil Kussat contributed at-sea support and preliminary analysis. We thank Akira Asada and Hiromi Fujimoto for their thoughtful reviews. We gratefully credit Richard Zimmerman, Dennis Rimington and Dave Price for engineering support and the Captain and crew of the R/V Roger Revelle. This work was supported by grants OCE9820026, OCE-9907247, and OCE-9819078 of the Marine Geology and Geophysics Program at NSF.

\section{References}

Caspary, W. F., Concepts of Network and Deformation Analysis, 183 pp., Monograph 11, Sydney, University of New South Wales, School of Surveying, 1988.

Chadwell, C. D., Shipboard towers for Global Positioning System antennas, Ocean Engineering, 30, 1467-1487, 2003.

Chadwell, C. D. and Y. Bock, Direst estimation of absolute precipitable water in ocean regions by GPS tracking of a coastal buoy, Geophys. Res. Lett., 28, 3701-3704, 2001.

Chen, C. T. and F. J. Millero, Speed of sound at high pressures, J. Acoust. Soc. Am., 62, 1129-1135, 1977.

Del Grosso, V. A., New equations for the speed of sound in natural waters (with comparisons to other equations), J. Acoust. Soc. Am., 56, 1084 1091, 1974.

Fujita, M., T. Ishikawa, M. Mochizuki, M. Sato, S. Toyama, M. Katayama, K. Kawai, Y. Matusumoto, T. Yabuki, A. Asada, and O. L. Colombo, GPS/Acoustic seafloor geodetic observations: method of data analysis and application, Earth Planets Space, 58, 265-275, 2006.

Gagnon, K., C. David Chadwell, and E. Norabuena, Measuring the onset of locking in the Peru-Chile Trench with GPS and acoustic measurements, Nature, 434, 205-208, 2005.

Hildebrand, J. A., C. D. Chadwell, S. M. Wiggins, and F. N. Speiss, Offshore geodetic monitoring on the southeast flank of Kilauea Volcano, Seismological Research Letters, 71, 232, 2000.

Kido, M., H. Fujimoto, S. Miura, Y. Osada, K. Tsuka, and T. Tabei, Seafloor displacement at Kumano-nada caused by the 2004 off Kii
Peninsula earthquakes, detected through repeated GPS/Acoustic surveys, Earth Planets Space, 58, 1-5, 2006.

Leick, A., GPS Satellite Surveying, 464 pp., John Wiley and Sons, 3rd edition, 2004.

Millero, F. J. and X. Li, Comments on "On equations for the speed of sound in seawater", J. Acoust. Soc. Am., 95, 2757-2759, 1995.

Miura, S., A. Sweeney, H. Fujimoto, H. Osaki, E. Kawai, R. Ichikawa, T. Konko, Y. Osada, and C. D. Chadwell, Evaluations of accuracy in kinematic GPS analyses using a precision roving antenna platform, EOS, 83, Fall Meet. Suppl. Abstract G52A-0959, 2002.

Obana, K., H. Katao, and M. Ando, Seafloor positioning system with GPSacoustic link for crustal dynamics observation-a preliminary result from experiments in the sea, Earth Planets Space, 52, 415-423, 2000.

Osada, Y., H. Fujimoto, S. Miura, A. Sweeney, T. Kanazawa, S. Nakao, S. Sakai, J. A. Hildebrand, and C. D. Chadwell, Estimation and correction for the effect of sound velocity variation on GPS/Acoustic seafloor positioning; an experiment off Hawaii Island, Earth Planets Space, 55, e17-e20, 2003.

Phillips, K. and C. D. Chadwell, Refined deformation models of the south flank of Kilauea Volcano, Hawaii based on seafloor geodetic data from 2000 to 2004, EOS, 86, Fall Meet. Suppl. Abstract G53B-0882, 2005.

Purcell, G. H., L. E. Young, S. K. Wof, T. K. Meehan, C. B. Duncan, S. S. Fisher, F. N. Spiess, G. Austin, D. E. Boegman, C. D. Lowenstein, C. Rocken, and T. M. Kelecy, Accurate GPS measurement of the location and orientation of a floating platform, Marine Geodesy, 14, 225-264, 1991.

Spiess, F. N., Suboceanic geodetic measurements, IEEE Transactions on Geosciences and Remote Sensing, 23, 502-510, 1985.

Spiess, F. N., C. D. Chadwell, J. H. Hildebrand, H. Dragert, D. Jabson, A. Sweeney, and R. Zimmerman, New geodetic reference stations on the Juan de Fuca Plate, EOS, 81, Fall Meet. Suppl. Abstract G11C-09, 2000.

Spiess, F. N., C. D. Chadwell, J. H. Hildebrand, L. E. Young, G. H. Purcell, Jr., and H. Dragert, Precise GPS/Acoustic positioning of seafloor reference points for tectonic studies, Phys. Earth Planet. Int., 108, 101-112, 1998.

Webb, F. H. and J. F. Zumberge, An introduction to GIPSY/OASIS-II, Technical Report D-11088, Jet Propulsion Lab, 1997.

Wilson, W. D., Equation for speed of sound in seawater, J. Acoust. Soc. Am., 32, 1357, 1960.

Yamada, T., M. Ando, K. Tadokoro, K. Sato, T. Okuda, and K. Oike, Error evaluation in acoustic positioning of a single transponder for seafloor crustal deformation measurements, Earth Planets Space, 54, 871-881, 2002 .

K. L. Gagnon and C. D. Chadwell (e-mail: cchadwell@ucsd.edu) 